\title{
Analysis of Linear Feedback Position Control in Presence of Presliding Friction
}

\author{
Michael Ruderman $^{* a)}$ Non-member, Makoto Iwasaki** Member
}

(Manuscript received April 10, 2015, revised Aug. 18, 2015)

\begin{abstract}
In various servo applications, the design of a precise positioning control constitutes a trade-off between the fast transient response and the short settling time for the required accuracy. Mostly, neither 'universal' control gains can be found to be equally suitable for both of these objectives. In this study, the design of a cascaded precise positioning control is analyzed in the presence of nonlinear friction. The nonlinear friction strongly impacts the reference settling and can deteriorate the positioning control performance. Two common cascaded structures, and that P-PI and PI-PI, are analyzed by exposing the closed-loop dynamics of the control system with friction in details. It is shown that a more 'traditional' integral control part is less useful when compensating for the nonlinear friction at the reference position settling. Furthermore, we show how the settling performance can be improved by applying a feed-forward friction observer. This can be included as a plug-in after designing the surrounding cascaded feedback control and without re-tuning. The proposed control strategy is evaluated experimentally on a standard industrial linear positioning axis, with a relatively high reference speed of $+/-500 \mathrm{~mm} / \mathrm{s}$ and narrow positioning error band of $+/-0.01 \mathrm{~mm}$.
\end{abstract}

Keywords: motion control, positioning, friction compensation, servo system, control design, observer

\section{Introduction}

A fast transient response and short settling time for the required error band are nearly always the opposite objectives for a precise positioning control in servo applications. In several applications such as data storage devices, machine tools, manufacturing tools for electronics components, and industrial robots the required specifications in motion performance, e.g. response and settling time and trajectory and settling accuracy, should be sufficiently achieved ${ }^{(1)}$. For instance, the settling control performance has been explicitly addressed in ${ }^{(2)}$ for the hard disk drives when using a dual actuator system. Mostly, neither 'universal' control gains can be found equally suitable for all the above mentioned control objectives. As a consequence, various gain-scheduling strategies (see e.g. ${ }^{(3)(4)}$ for survey) are often used in the industrial applications, in particular when designing the inner velocity control loop. However, these can be cumbersome in the design and stability analysis, and often involve several ad-hoc steps. Among other related challenges, the linearization gain scheduling depends on intuitive rules of thumb and extensive simulations for evaluating the control stability and performance $^{(4)}$. For instance, a two-step controller for fast and precise positioning of a X-Y table has been reported in ${ }^{(5)}$. Two PID controllers have been designed separately, once for the fast move in a macro-dynamic range, and once for the settling within a micro-dynamic range. The switching condition

a) Correspondence to: Michael Ruderman. E-mail: michael. ruderman@uia.no

* University of Agder

Jon Lilletunsvei 9, Grimstad, 4879, Norway

** Nagoya Institute of Technology

Gokiso, Showa-ku, Nagoya 466-8555, Japan between two controllers has been determined by a method based on the energy conservation. Apart from the above mentioned gain scheduling, a robust 2-DOF control ${ }^{(6)}$, which proved to be efficient for rejecting the force/torque disturbances and similarly for coping with unknown/variable loads, can be applied when designing the inner velocity loop.

Apart from the residual vibrations, which appear in multimass systems and are out of scope in this work, the settling response of a positioning control is mostly affected by the nonlinear presliding friction. The latter is characterized by appearance of the hysteresis in displacement and that after each motion reversal (see ${ }^{(7)}$ for details on friction dynamics). Several strategies have been proposed in the past to overcome frictional nonlinearities in the motion control. Simplest way, a pure model-based feed-forward friction compensation can be added to a standard linear feedback control. e.g. PD one. Though this allows attaining the accurate position tracking results, such compensation strategy lacks generally on the robustness against the friction uncertainties and time-varying behavior. Another control of systems with dynamic friction, based on the gain scheduling, has been proposed in ${ }^{(8)}$, where a gain-scheduled linearized friction estimator has been combined with a PI velocity control. Here the given reference velocity appears as the scheduling variable. However, the simulation results only have been shown, and the overall control design is based on the assumption that the dynamic friction complies with a LuGre-modeled behavior, that in various real applications can be highly different case. An efficient adaptive friction compensation proposed in ${ }^{(9)}$ can be also used for the position control, however, assuming the friction uncertainties related basically to the Coulomb friction coefficient only. This approach is also not accounting for pre-sliding hysteresis curves and assumes a modeled discontinuity at 
zero crossing velocity. A PID tuning approach aimed for suppressing the stick-slip behavior caused by the friction in transmission systems has been presented in ${ }^{(10)}$. The method uses the describing function analysis and pole-placement for deriving a sequence of ad-hoc design rules which allow the controller to achieve a fast response without inducing the limit cycles. However, no pre-sliding friction range with a consequent residual 'creeping' response, due to the integral control action, has been explicitly addressed. Another possible way to compensate for the presliding friction is to observe explicitly the corresponding presliding state(s) and to inject this (these) into the control signal, as proposed in ${ }^{(11)(12)}$ and recently in ${ }^{(13)}$. The problem of position settling performance in presence of the nonlinear friction has been also addressed in ${ }^{(14)}$. Here a split initial value compensation has been used for the mode-switching control in vicinity to the target position.

Even if it is not immediately evident a traditional use of the integral control term cannot not improve the position settling response in presence of the nonlinear friction. On the contrary, the so-called hunting limit cycles can be induced through the integral control action, and the linear feedback control tends to micro-oscillations around the target position. This phenomenon of friction-induced hunting limit cycles has been explicitly addressed in ${ }^{(15)}$, while taking the LuGre and static switch friction models for analysis. Also the hysteresis-related damping due to presliding friction in vicinity to the motion stop has been recently analyzed in the literature, e.g. for the LuGre and Maxwell-slip friction models. However, to the best of our knowledge, the related problems of positioning settling have not been fully explored up to the present, above all due to the complex and not fully understood nonlinear presliding mechanisms acting on the contact interface in vicinity to zero velocity.

The aim of this paper is to analyze the settling behavior of the common positioning controls with cascaded P-PI or PI-PI structures, and that in presence of nonlinear friction. Furthermore, we demonstrate a possible way of compensating the nonlinear friction, by adding the feed-forward friction observer (FFFO) which has been proposed and elaborated in ${ }^{(11)}$. It is worth noting here that the recent work addresses the feedback control loop only, that is without considering the feed-forward control action (see ${ }^{(1)}$ for details on the 2-DOF precision control). As implication, a transient overshoot in the recent positioning is deliberately allowed. A preliminary version of the following results have been presented at IPECHiroshima 2014 conference $^{(16)}$.

The rest of the paper is organized as follows. The main part with analysis of the closed loop dynamics and settling behavior in presence of the nonlinear friction is given in Sect. 2. Section 3 describes the experimental system of linear positioning axis used in this work. The structure of the cascaded positioning control with FFFO compensator is explained in Sect. 4. Section 5 shows the experimental control evaluation. Concluding remarks are given in Sect. 6.

\section{Analysis of Settling Behavior}

Consider a simple one degree-of-freedom motion system described by the following differential equation

$$
m \ddot{x}(t)=u(t)-F(\dot{x}(t), z(t)), \cdots \cdots \cdots \cdots \cdots \cdots(1)
$$

where $m$ is the moving mass and $F$ is the friction. Assume that the latter constitutes a dynamic nonlinear map of the relative velocity $\dot{x}$ and internal state $z$ which represents a relative displacement after each motion reversal. It is worth noting that the assumed friction function complies with the generalized phenomenological friction model structure established in ${ }^{(7)}$. We also make aware that the friction $F(\cdot)$ does not include the linear (viscous) friction term. The latter constitutes solely a linear damping contribution and thus enters the velocity gain of the feedback control loop. Further, assume that the system is forced by a scalar control value $u$ which is the output of the inner (velocity) feedback control loop.

2.1 Presliding Friction Dynamics Now let us examine the friction dynamics in more details, in particular in a range which is close to the controlled position settling, that means controlled motion stop. Taking the total friction derivative with respect to the time one can easily obtain

$$
\dot{F}(\dot{x}, z)=\frac{\partial F}{\partial \dot{x}} \ddot{x}+\frac{\partial F}{\partial z} \dot{x} . \ldots \ldots \ldots \ldots \ldots \ldots \ldots
$$

Note that due to the relative presliding distance $z$ it is valid $\dot{z}=\dot{x}$. It can be seen that even if the first right-hand side term in (2) can be neglected in presliding, the second right-hand side term remains significant at non-zero velocities. That means within a settling phase the impact of friction derivative contributes substantially to the overall system dynamics. Note that the impact becomes even larger, closer the system is to the motion reversal state. The latter can be explained by means of presliding friction curves which are schematically shown in Fig. 1. Recall that the indicated bound between the presliding and sliding is not explicitly defined and is rather characterized by zero friction dynamics (see ${ }^{(17)}$ for details).

In general, it is rarely to expect that a fast feedback positioning control provides zero transient overshoot at a large moving distance with relative high velocities and accelerations. Otherwise, an under-determined control would lead to an untimely motion stop which has a similar impact of the presliding friction. That is because a novel motion onset should be enforced, after untimely stop, for reaching the required reference position. Generally, we assume that a transient overshoot is present even if it is infinitesimal small, however beyond the sensing resolution of the feedback control. That means an inevitable motion reversal occurs, after which the friction curve undergoes a nonlinear hysteresis pathway as exemplarily shown in Fig. 1. It is evident that

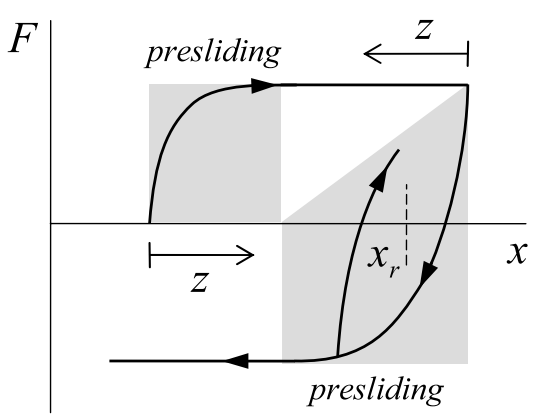

Fig. 1. Presliding friction curves at motion onset and motion reversals 
within presliding the $\partial F / \partial z$ term is considerable and above all non-constant. To that end, one can recognize that the disturbing contribution of the friction dynamics (2) cannot be considered as a function of time, but rather as that of the instantaneous distance to the reference state.

2.2 P-PI Control Loop Dynamics First we analyze the P-PI cascaded structure, while the inner PI velocity loop is surrounded by the outer $\mathrm{P}$ position loop. Substituting the standard PI velocity control

$$
u(t)=K_{p}\left(\dot{x}_{r}(t)-\dot{x}(t)\right)+K_{i} \int\left(\dot{x}_{r}(t)-\dot{x}(t)\right) d t
$$

and $\mathrm{P}$ positioning control

$$
\dot{x}_{r}(t)=P\left(x_{r}(t)-x(t)\right) \cdot
$$

into (1) one obtains the closed-loop dynamics of the total cascaded control system as

$$
\begin{aligned}
m \ddot{x}+ & K_{p} \dot{x}+\left(K_{i}+P K_{p}\right) x+P K_{i} \int x d t \\
& +F(\dot{x}, z)=P\left(K_{p} x_{r}+K_{i} \int x_{r} d t\right) .
\end{aligned}
$$

Note that in (5), and further on, we omit the time argument for the sake of simplicity. Taking the time derivative of (5) and assuming $\dot{x}_{r}=0$ for the reference, this due to positioning task we are interested in, one obtains the control system dynamics as

$$
m \dddot{x}+K_{p} \ddot{x}+\left(K_{i}+P K_{p}\right) \dot{x}+P K_{i} x+\dot{F}(\dot{x}, z)=P K_{i} x_{r} .
$$

Reducing the friction dynamics to the second right-hand side term of (2), as explained in Sect. 2.1, we obtain

$$
m \dddot{x}+K_{p} \ddot{x}+\left(K_{i}+P K_{p}+\frac{\partial F}{\partial z}\right) \dot{x}+P K_{i} x=P K_{i} x_{r} .
$$

It can be seen that the steady-state accuracy, and that with the specified transient, can be achieved in case of zero or linear friction dynamics only. Note that the former $(\partial F / \partial z=0)$ corresponds to the constant Coulomb friction, while latter $(\partial F / \partial z=$ const $)$ constitutes the linear (viscous) friction effect. Neither of both represent the general case of the nonlinear friction in presliding, as it can be perceived from Fig. 1. In order to analyze the eigendynamics of (7), and in particular to show the control stability, consider the related control system dynamics in the following form

$$
\dddot{x}+a_{1} \ddot{x}+a_{2}(t) \dot{x}+a_{3} x=0,
$$

where

$$
a_{1}=\frac{K_{p}}{m}, a_{2}=\frac{1}{m}\left(K_{i}+P K_{p}+\frac{\partial F}{\partial z}\right), a_{3}=\frac{P K_{i}}{m} .
$$

Applying the Routh's stability criterion (see e.g. ${ }^{(18)}$ ) one can show that the first column of the Routh array is

$$
\left[1, \frac{K_{p}}{m}, \frac{1}{m}\left(K_{i}+P K_{p}+\frac{\partial F}{\partial z}\right)-\frac{P K_{i}}{K_{p}}, \frac{P K_{i}}{m}\right]^{T},
$$

which is required to have all elements positive. Since $\partial F / \partial z \geq 0$ and all the control gains, as well as the moving mass, are assumed to be positive the single condition that should hold is

$$
\frac{K_{p}}{P}+\frac{K_{p}^{2}}{K_{i}}>m
$$

This can be easily ensured when designing the feedback control gains after having estimated the moving mass.

In order to analyze the impact of $\partial F / \partial z$ on the closed loop dynamics we apply the root locus method of Evans (see e.g. ${ }^{(18)}$ for details). For the sake of simplicity we assume $m=1$, this for the rest of the subsection, and rewrite (8) as

$$
1+K \frac{b(s)}{a(s)}=0
$$

where

$$
K=\partial F / \partial z, \quad b(s)=s, \quad a(s)=s^{3}+a_{1}^{*} s^{2}+a_{2}^{*} s+a_{3}^{*},
$$

with the polynomial coefficients

$$
a_{1}^{*}=K_{p}, \quad a_{2}^{*}=K_{i}+P K_{p}, \quad a_{3}^{*}=P K_{i} .
$$

This allows directly to illustrate the root locus of the closedloop control system in dependency of $K \in[0, \infty)$, i.e. of the presliding friction state. For an illustrative example, we assign the controllable poles of the closed-loop to $\lambda_{1,2,3}=$ $[-10,-20,-30]$, that yields the characteristic polynomial coefficients $a_{1}^{*}=60, a_{2}^{*}=1100, a_{3}^{*}=6000$.

The resulted root locus diagram is shown in Fig. 2. It can be seen that with an increasing $\partial F / \partial z$ value the dominant pole, which determines the transient of the controlled position, moves towards zero, thus inherently slowing down the control response. At the same time, larger $\partial F / \partial z$ values give rise to the conjugate-complex pole pair with the imaginer part going towards infinity as $\partial F / \partial z \rightarrow \infty$. This is inherently attributed to an increasing stiffness of the contact (presliding) friction, i.e. immediately after a motion reversal.

From the analysis made above one can conclude that closer the motion onset or motion reversal to the reference (settling) position is, more deteriorated is the control performance due to the nonlinear presliding friction.

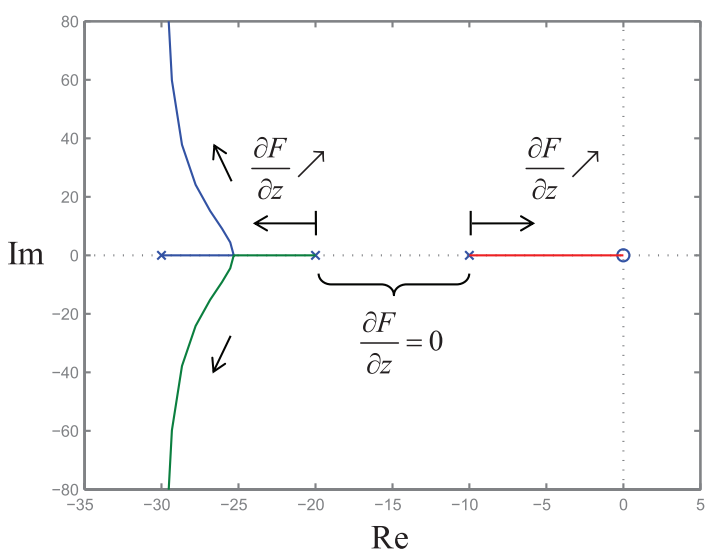

Fig. 2. Root locus diagram of closed control loop in dependency of $\partial F / \partial z$ 
2.3 PI-PI Control Loop Dynamics When connecting the outer PI positioning control

$$
\dot{x}_{r}=P\left(x_{r}-x\right)+I \int\left(x_{r}-x\right) d t, \cdots \cdots \cdots \cdots \cdots
$$

that instead of (4), to the same (inner) velocity loop (3) one obtains the overall closed-loop dynamics as

$$
\begin{aligned}
m \dddot{x} & +K_{p} \dddot{x}+\left(P K_{p}+K_{i}\right) \ddot{x}+\left(I K_{p}+P K_{i}\right) \dot{x} \\
& +I K_{i} x+\ddot{F}(\dot{x}, z)=r\left(\ddot{x}_{r}, \dot{x}_{r}, x_{r}\right) . \cdots \cdots \cdots \cdots
\end{aligned}
$$

Note that the right-hand side of (12)

$$
r\left(\ddot{x}_{r}, \dot{x}_{r}, x_{r}\right)=P K_{p} \ddot{x}_{r}+\left(I K_{p}+P K_{i}\right) \dot{x}_{r}+I K_{i} x_{r}
$$

contains only the reference-based excitation of the closedloop dynamics. Here again, one can recognize that the nonlinear friction term disturbs the convergence of the controlled position towards the reference value. Important to note is that in (12) the second-order friction term has to be considered. That means no frictional impact on the closed-loop behavior can be expected if and only if the presliding friction dynamics complies $\dot{F}=$ const. This is, however, generally not a case as has been demonstrated by means of the hysteresis curves shown in Fig. 1.

In order to analyze the impact of presliding friction dynamics consider

$$
\ddot{F}(\dot{x}, z)=\frac{d}{d t} \dot{F}(\dot{x}, z)=\frac{\partial F}{\partial \dot{x}} \dddot{x}+\frac{\partial F}{\partial z} \ddot{x} . \cdots \cdots \cdots \cdots
$$

It is evident that even if the first right-hand side term in (14) can be neglected, this for the same reason as described in Sect. 2.2, the second right-hand term remains substantial at the motion onsets and motion reversals. When substituting $\partial F / \partial z \ddot{x}$ into the closed-loop dynamics (12) the similar analysis of the Routh's stability and root locus, as provided in Sect. 2.2, can be accomplished without principal discrepancies. This is, however, omitted for the sake of clarity and comprehensibility. Instead, we are to analyze the principle low-order dynamics of (12), while assuming a stable parameterization of the closed-loop control system.

When neglecting the higher-order terms in (12) the eigendynamics of the closed control loop can be approximated by

$$
\left(P K_{p}+K_{i}+\frac{\partial F}{\partial z}\right) \ddot{x}+\left(I K_{p}+P K_{i}\right) \dot{x}+I K_{i} x=0 .
$$

It can be easily seen that (15) constitutes the second-order system dynamics of the form

$$
c(z) \ddot{x}+d \dot{x}+k x=0, \cdots
$$

where the stiffness $k$ and damping $d$ are determined by the corresponding control gains. At the same time, the inertia $c$ is not longer constant and depends on the relative displacement $z$ within the instantaneous presliding range. Recall that ones the nonlinear friction saturates the $\partial F / \partial z$ term becomes zero and also the inertial term becomes constant $c_{0}=K_{p} P+K_{i}$. However, the inertial term $c(z)$, i.e. during presliding, cannot be fully determined by the control gain selection only. This has a substantial impact on the position control performance as will be exposed in the following by analyzing the $(x, \dot{x})$ trajectories of (16). Recall that (16) represents the simplified dynamics of the closed-loop control system which allows us, however, to demonstrate qualitatively the principal impact of presliding friction.

2.4 Simulation of Motion Trajectories at Controlled Settling Now, let us analyze and compare the trajectories of simplified control system (16), once with and once without impact of presliding friction. The following control parameters are assumed for a numerical simulation: $K_{p}=0.01$, $K_{i}=0.1, P=100$, and $I=1000$. Note that the selected control parameters, excepting the integral positioning term $I$, are the same as are used in the experimental evaluation described further in Sect. 5. The integral position term is omitted in the experimental evaluation. The simulated presliding term is assumed to be invers to the relative displacement, so that $\partial F / \partial z \sim z^{-1}$. Here it is worth noting that another functional maps, e.g. exponential one, are equally thinkable for representing $\partial F / \partial z$ which should comply with the presliding hysteresis curves. Most important is that $\partial F / \partial z$ possesses a relatively high value, or even $\partial F / \partial z \rightarrow \infty$ at $z \rightarrow 0$, and is rapidly decreasing afterwards as $z$ passes a relatively small presliding distance. Since (16) constitutes a free system, its dynamic response is simulated by setting an initial value $x(0) \neq 0$. Recall that this is equivalent to applying a constant position reference. Two cases are considered $x(0)=0.1$ and $x(0)=0.01$ that means a micro-positioning with the relative displacements which are differing by one order of magnitude. The $(x, \dot{x})$ trajectories are shown in Fig. 3 for both cases. It can be seen that for $\partial F / \partial z=0$ a pure linear behavior results in the direct acceleration and deceleration phases without any position overshoots. On the contrary, when $\partial F / \partial z \neq 0$, several oscillating cycles occur before the trajectory approaches the equilibrium state. Further, it can be seen that the relative (percental) positioning overshoot becomes even larger when the relative displacement decreases, i.e. in case $x(0)=0.01$. At the same time, the reached velocity magnitude decreases drastically, which results in a clearly slower final positioning. The latter becomes evident when inspecting the corresponding time series of the controlled position shown in Fig. 4. For both relative displacements, i.e. $x(0)=0.1$ and $x(0)=0.01$, the linear case $(\partial F / \partial z=0)$ provides a fast exponential convergence to the equilibrium state. In case $\partial F / \partial z \neq 0$, the control system exhibits a long-term nonlinear oscillation pattern, obviously due to the impact of presliding friction. It should be stressed that the convergence time increases dramatically
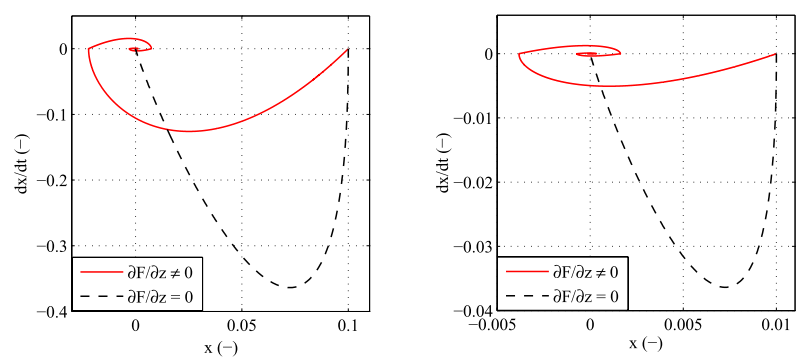

Fig. 3. Simulated trajectories of system (16) with $(\partial F / \partial z \neq 0)$ and without $(\partial F / \partial z=0)$ impact of presliding friction 

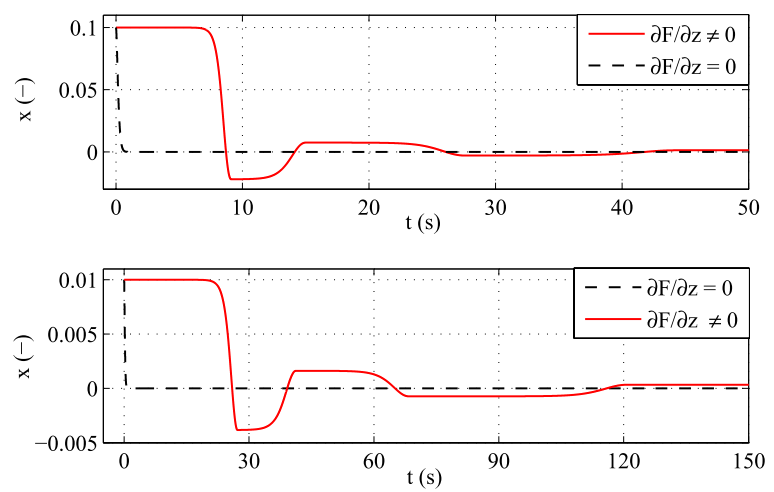

Fig. 4. Simulated position settling of system (16) with $(\partial F / \partial z \neq 0)$ and without $(\partial F / \partial z=0)$ impact of presliding friction

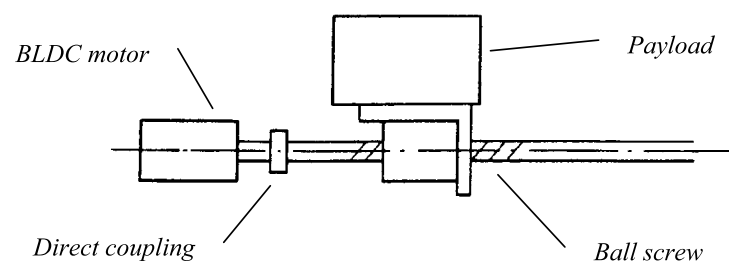

Fig. 5. Schematic representation of linear axis system

Table 1. Technical specifications of linear axis system

\begin{tabular}{lll}
\hline maximal motor torque & 1.9 & $\mathrm{Nm}$ \\
rated motor torque & 0.64 & $\mathrm{Nm}$ \\
rated motor current & 1.5 & $\mathrm{~A}$ \\
ball-screw pitch lead & 20 & $\mathrm{~mm}$ \\
maximal speed of table drive & 1480 & $\mathrm{~mm} / \mathrm{s}$ \\
maximal stroke of table drive & 400 & $\mathrm{~mm}$ \\
\hline
\end{tabular}

by the decreased positioning distance. The flat extrema of oscillating pattern indicate the appearance of the stick-slip behavior, and that in view of the integral control action.

From the numerical analysis described above it can be concluded that smaller the considered micro-positioning range is, less efficient is the linear feedback regulation, even in presence of the integral control terms. Furthermore it appears obvious that an incorporation of integral control term into the outer position loop does not bring any improvements for compensation of the nonlinear presliding friction during the reference position settling.

\section{Linear Axis System}

The linear axis system used is this work is schematically represented in Fig. 5. This constitutes a standard industrial linear guide actuator based on the ball-screw transmission. The moving carrier with a payload is actuated by the BLDC (brushless direct current) motor which is directly coupled to the ball-screw shaft. The key data of the system are taken from the technical specification and listed in Table 1. The angular motor displacement and corresponding angular velocity are captured by the motor-embedded 16-bit serial encoder connected to the motor drive amplifier. Accordingly, the theoretical sensor-based resolution of the linear motion is about $0.3 \mu \mathrm{m}$. However, the real positioning repeatability is limited by the mechanical backlash in ball-screw, which nominal

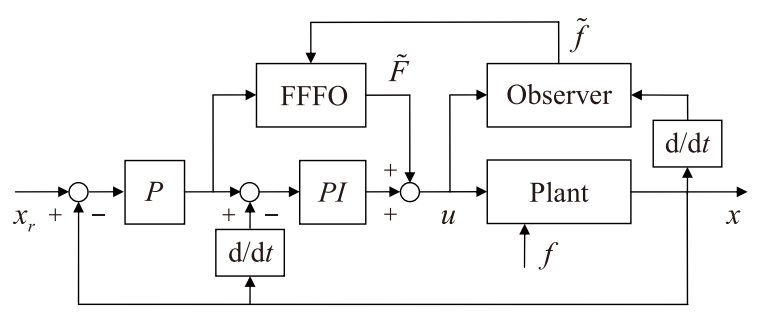

Fig. 6. Block diagram of cascaded positioning control with FFFO

value accounts for $20 \mu \mathrm{m}$. The reference motor torque, provided to the standard analog interface of the motor drive amplifier, constitutes the output of the inner velocity control loop. The control implementation and evaluation are performed using the DS1104CLP dSpace realtime board with the sampling time set to $500 \mu \mathrm{s}$.

\section{Positioning Control}

4.1 Control Structure The applied positioning control is shown in Fig. 6. The controller constitutes a common cascaded P-PI structure extended by the FFFO aimed to compensate for the nonlinear dynamic friction. Note that the FFFO is driven (see ${ }^{(1)}$ for details) by the output of position control, which in turn constitutes the velocity reference value. The observed friction disturbance $\tilde{f}$, which is used by the FFFO, is provided by the observer whose inputs are the control value $u$ and measured relative velocity $\dot{x}$. Examining the control structure it becomes evident that the applied FFFO scheme can be easily plugged-in to the common cascaded control, which can be designed separately. This appears as particularly suitable for several industrial applications, where the standard PI-type cascaded controls represent a common solution implemented on the dedicated hardware.

4.2 Feed-Forward Friction Observer (FFFO) The feed-forward friction observer (FFFO), first proposed in ${ }^{(19)}$ and elaborated in ${ }^{(11)}$, allows to combine both, the modelbased friction feed-forwarding and observer-based estimation of the friction-related disturbances. In the following, the applied FFFO is briefly summarized for convenience of the reader. For more details, however, we refer to ${ }^{(11)(19)}$.

The unknown time-variant friction term $f(t)$ can be assumed as an input disturbance so that the plant dynamics will be captured by

$$
m \ddot{x}(t)+F(\dot{x}(t))+f(t)=u(t) \ldots \ldots \ldots \ldots \ldots \ldots(t)
$$

After determining the plant description, i.e. $\tilde{m}$ and $\tilde{F}(\cdot)$ terms $^{\dagger}$, the friction-related disturbance can be observed by

$$
\tilde{f}(t)=u(t)-\tilde{m} \ddot{x}(t)-\tilde{F}(\dot{x}(t)) \text {. }
$$

It is evident that the computed disturbance observation will equally contain the (spurious) high-frequent components, which are mainly due to the time derivative of the measured output quantities and high-order harmonics which are not captured by the simplified model (17). However, these highfrequent dynamic components are not interfering the output

$\dagger$ Here the dynamic friction, and that in the input-output notation, is explicitly denoted as a function of relative velocity only. The $z$ argument, as in (1), is deliberately skipped since this constitutes an internal (not available) friction state. 
of FFFO because of the inherent low-pass characteristics of the latter, as explained in details in ${ }^{(11)}$.

The feed-forward friction observer is given by

$$
\dot{\tilde{F}}(t)+\frac{B\left|\dot{x}_{i n}\right|}{\left|S\left(\dot{x}_{i n}\right)\right|} \tilde{F}(t)=\dot{\tilde{z}}_{1}(t)+B \dot{x}_{i n}+L \tilde{f}(t) . \cdots \cdots
$$

Here, the attributes of 2SEP dynamic friction model (see ${ }^{(11)(17)}$ for details) are the weighting parameter $B$ of the time constant, dynamic presliding friction state $\tilde{z}_{1}$, and the static Stribeck characteristic curve $S(\cdot)$. Note that the input velocity value $\dot{x}_{i n}$ depends on the placement of FFFO within the control loop. Here it constitutes the output of P-control, according to Fig. 6.

According to ${ }^{(11)}$ the observation gain

$$
L=\frac{B\left|\dot{x}_{i n}\right|}{\left|S\left(\dot{x}_{i n}\right)\right|}
$$

brings the right-hand side excitation of observer on the same scale as the output friction force (cf. left- and right-hand sides in (19)), thus ensuring the steady-state of observer as

$$
\tilde{F}=S\left(\dot{x}_{i n}\right)+\tilde{f},
$$

after the transient response. Note, that the observation gain $L$ is computed analytically and does not require any design parameters to be selected. For further details on the FFFO properties the reader is refereed to ${ }^{(11)}$.

4.3 Linear Feedback Control The linear feedback control, i.e. the P-PI one, is designed in two consecutive stages, starting by the inner velocity loop and following by the outer positioning loop. Having a linear plant approximation $G(j \omega)=\dot{X}(j \omega) / U(j \omega)$, which is simple case a first-oder time delay transfer element, the PI velocity control gains can be easily assigned using e.g. the pole-placement, bode diagrams, and other convenient techniques of the classical control theory ${ }^{(18)}$. Note, that besides the actuator limits the admissible control gains can be bounded by the impact of deadtime (see e.g. ${ }^{(20)}$ for details), which is frequently encountered in the digital control systems. Recall, that a possibly large selection of the integral control gain argues for a better cancelation of the steady-state friction, but not necessarily of the presliding friction. After designing and evaluating the inner velocity loop an appropriate position control gain $\mathrm{P}$ can be easily selected taking into account (i) the maximal relative velocity of the plant, and (ii) acceptable control overshoot before settling. It is worth to recall that an additional integral term in the outer positioning loop does not improve the compensation of the nonlinear friction as has been analyzed in Sect. 2.

\section{Experimental Evaluation}

The following experimental results provide evidence of the limited performance of the linear cascaded feedback control, including the integral term, in presence of pre-sliding friction close to the controlled motion stop. The settling behavior at the reference position contains the signature of nonlinear pre-sliding friction for which the overall motion dynamics changes, as analyzed in Sects. 2.2 and 2.3.

The designed P-PI cascaded control and the same cascaded control extended by the FFFO (see the block diagram in Fig. 6) have been experimentally evaluated on the linear axis
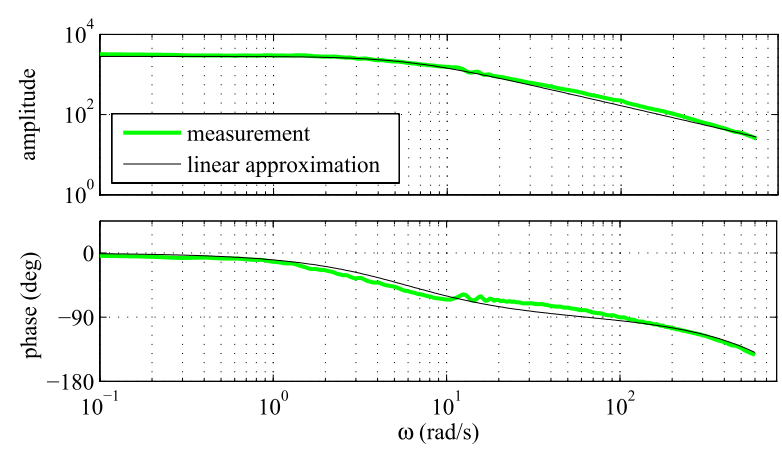

Fig. 7. Measured frequency response function and linear plant approximation $G$ at higher excitation amplitude

described in Sect. 3. Before the control design and evaluation, the system has been identified once in order to determine the friction model parameters used for the FFFO, and once in order to obtain a linear plant approximation required for tuning the feedback control gains. Two different motion experiments have been accomplished to identify the system parameters. First, a low-gain closed loop position tracking has been performed so as to determine the Coulomb friction level and initial hysteresis stiffness of the 2SEP friction model (see ${ }^{(17)}$ for details). Second, the frequency-response measurements at different excitation levels have been done in the range $1-100 \mathrm{~Hz}$ so as to determine the residual nonlinear and linear system parameters. For more details on the frequencydomain system identification in presence of nonlinear friction we refer to ${ }^{(21)}$. The measured frequency response function at higher excitation amplitude ${ }^{\dagger}$ is exemplarily shown in Fig. 7 versus the linear plant approximation $G(j \omega)$. The determined feedback control parameters are $K_{p}=0.01, K_{i}=0.1$, and $P=100$. Note that the P-PI control parameters have been determined in accord with a design procedure described in Sect. 4.3.

The motion profile shown in Fig. 8 (above) has been taken for the control evaluation. The reference trajectory constitutes the bidirectional positioning at different distances with the constant velocity set to $\pm 500 \mathrm{~mm} / \mathrm{s}$. Note, that the latter has been selected so as to protect the mechanical structure of linear axis system (compare with the data in Table 1). Two independent experiments, and that with different initial positions, have been repeated, each one when using the P-PI and then P-PI-FFFO controller. It is worth noting that each of the experiments is starting from another initial (absolute) position of the linear axis, i.e. state of the ball-screw. This yields quite differing initial system states, thus showing how insensitive is the control scheme with respect to the positionvarying friction.

The close-ups of settling behavior at the $250 \mathrm{~mm}$ and $75 \mathrm{~mm}$ references (see Fig. 8 in the middle and below) disclose the inferior performance of the P-PI positioning control. The indicated error band of $\pm 0.01 \mathrm{~mm}$ relates to the nominal backlash of the ball-screw (see Sect. 3) and can be assumed as the required upper bound of the positioning accuracy. It is evident that a slow settling after the transient

\footnotetext{
$\dagger$ Note that higher the excitation level is, lower is the impact of nonlinear friction (see e.g. ${ }^{(11)}$ ). Therefore, the sufficiently excited frequency response of the system with friction nonlinearities approaches the corresponding linear transfer function characteristics.
} 

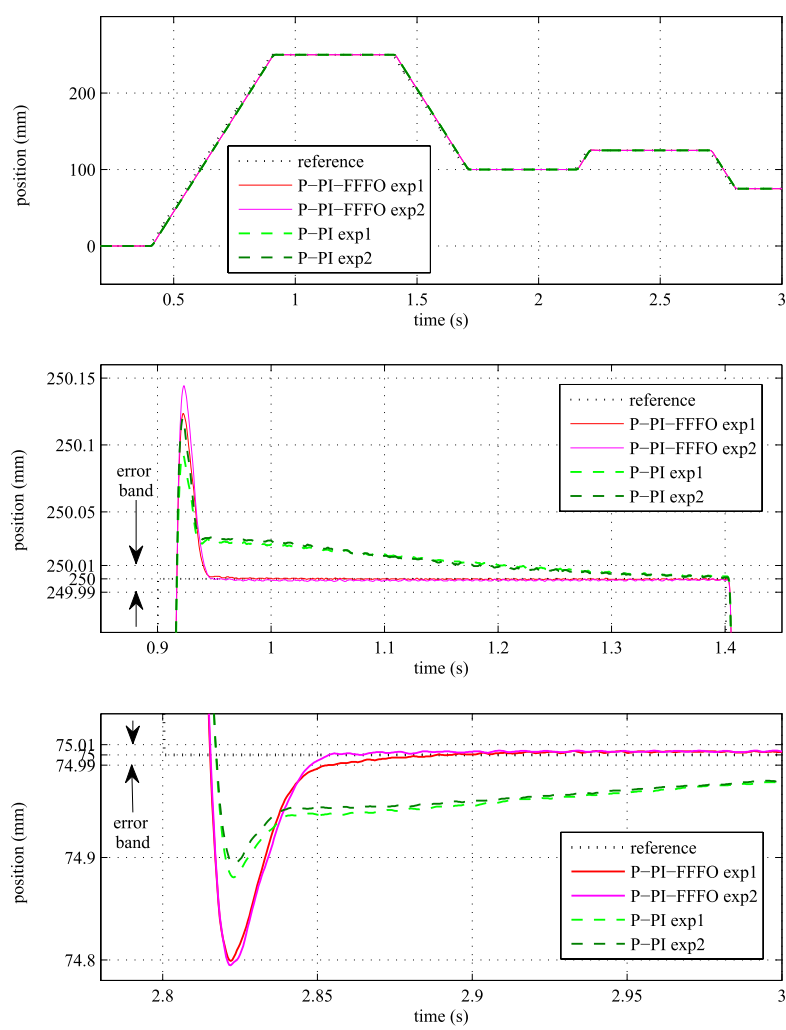

Fig. 8. Experimental positioning response using P-PI and P-PI-FFFO controllers. The overall positioning trajectory above and close-ups of settling response in the middle and below

overshoot relates to the nonlinear friction in presliding and cannot be efficiently compensated by a linear feedback control only, as has been discussed before in Sect. 2. On the contrary, the P-PI-FFFO control is free of the slow settling and, at the same time, has only a slightly higher overshoot than its P-PI competitor. Further it can be noted that the P-PI control response in the range where an overshoot peak changes to a slow (creeping) convergence behavior can be seen as a signature of the instantaneous presliding region, within which the nonlinear friction deteriorates the linear control behavior.

\section{Conclusions}

In this paper, we have analyzed the settling behavior of a precise positioning control in presence of nonlinear friction. The P-PI and PI-PI cascaded control structures, which constitute the common practice in multiple servo applications, have been considered. Taking into account the generic presliding friction dynamics, i.e. without assuming any particular friction model, the closed-loop control loop behavior has been analyzed for both cases P-PI and PI-PI. By deriving the corresponding differential equations with the variable, i.e. frictiondependent, coefficients we have exposed the impact of nonlinear friction on the positioning control performance.

It has been shown by theoretical analysis and proved with experiments that the traditional integral control action is less efficient when compensating for the nonlinear presliding friction close to the reference position settling. This gives us a reasoning for omitting design of an appropriate integral control part, which can be often circumstantial, in favor of alternative, e.g. model-based, friction compensators. Using the FFFO-based compensator, plugged-in to the existing cascaded structure, we have demonstrated a significant improvement in the positioning control performance and that without changing/retuning the surrounding control loop.

The experimental control evaluation has been accomplished on an industrial linear positioning axis with the standard interfaces for the input reference torque and output encoder position, both available in the motor amplifier.

\section{References}

( 1 ) M. Iwasaki, K. Seki, and Y. Maeda: "High-precision motion control techniques: A promising approach to improving motion performance", IEEE Industrial Electronics Magazine, Vol.6, No.1, pp.32-40 (2012)

( 2 ) H. Numasato and M. Tomizuka: "Settling control and performance of a dualactuator system for hard disk drives", IEEE/ASME Transactions on Mechatronics, Vol.8, No.4, pp.431-438 (2003)

( 3 ) K. Astrom, T. Hagglund, C. Hang, and W. Ho: "Automatic tuning and adaptation for PID controllers-a survey", Control Engineering Practice, Vol.1, No.4, pp.699-714 (1993)

( 4 ) W.J. Rugh and J.S. Shamma: "Research on gain scheduling", Automatica, Vol.36, No.10, pp.1401-1425 (2000)

( 5 ) Y.-T. Tseng and J.-H. Liu: "High-speed and precise positioning of an x-y table", Control Engineering Practice, Vol.11, No.4, pp.357-365 (2003)

( 6 ) T. Umeno and Y. Hori: "Robust speed control of DC servomotors using modern two degrees-of-freedom controller design", IEEE Transactions on Industrial Electronics, Vol.38, No.5, pp.363-368 (1991)

( 7 ) F. Al-Bender and J. Swevers: "Characterization of friction force dynamics", IEEE Control Systems Magazine, Vol.28, No.6, pp.64-81 (2008)

( 8 ) C. Vivas, F. Rubio, and C. Canudas-De-Wit: "Gain-scheduling control of systems with dynamic friction", in IEEE 41st Conference on Decision and Control, Vol.1, pp.89-94 (2002)

( 9 ) B. Friedland and Y.-J. Park: "On adaptive friction compensation”, IEEE Transactions on Automatic Control, Vol.37, No.10, pp.1609-1612 (1992)

(10) C.-C. Cheng and C.-Y. Chen: "A PID approach to suppressing stick-slip in the positioning of transmission mechanisms", Control Engineering Practice, Vol.6, No.4, pp.471-479 (1998)

(11) M. Ruderman: "Tracking control of motor drives using feed-forward friction observer (FFFO)", IEEE Transactions on Industrial Electronics, Vol.61, No.7, pp.3727-3735 (2014)

(12) M. Ruderman and M. Iwasaki: "Control of pre-sliding friction using nonlinear state observer", in IEEE 13th International Workshop on Advanced Motion Control (AMC), pp.592-597 (2014)

(13) M. Ruderman and M. Iwasaki: "Observer of nonlinear friction dynamics for motion control", IEEE Transactions on Industrial Electronics, Vol.62, No.9, pp.5941-5949 (2015)

(14) Y. Maeda, M. Wada, M. Iwasaki, and H. Hirai: "Improvement of settling performance by mode-switching control with split initial-value compensation based on input shaper", IEEE Transactions on Industrial Electronics, Vol.60, No.3, pp.979-987 (2013)

(15) R.H.A. Hensen, M.J.G. Van de Molengraft, and M. Steinbuch: "Friction induced hunting limit cycles: A comparison between the LuGre and switch friction model", Automatica, Vol.39, No.12, pp.2131-2137 (2003)

(16) M. Ruderman and M. Iwasaki: "Analysis of settling behavior and design of cascaded precise positioning control in presence of nonlinear friction", in International Power Electronics Conference (IPEC-Hiroshima 2014 - ECCEASIA), pp.1665-1670 (2014)

(17) M. Ruderman and T. Bertram: "Two-state dynamic friction model with elasto-plasticity", Mechanical Systems and Signal Processing, Vol.39, No.12, pp.316-332 (2013)

(18) G.F. Franklin, J.D. Powell, and E.A. Naeini: Feedback Control of Dynamic Systems, 6th ed. Prentice Hall (2009)

(19) M. Ruderman and T. Bertram: "Feed-forward friction observer (FFFO) for high-dynamic motion control", in IEEE 20th Mediterranean Conference on Control and Automation, pp.1013-1018 (2012)

(20) M. Ruderman and T. Bertram: "Variable proportional-integral-resonant (PIR) control of actuators with harmonic disturbances", in IEEE International Conference on Mechatronics (ICM), pp.846-851 (2013)

(21) M. Ruderman and T. Bertram: "FRF based identification of dynamic friction using two-state friction model with elasto-plasticity", in IEEE International Conference on Mechatronics (ICM), pp.230-235 (2011) 
Michael Ruderman (Non-member) received the B.Sc. degree in ap-

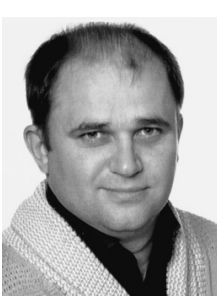
plied physics from the Polytechnical University of Kharkov, Kharkov, Ukraine, in 1997 and the Dipl.Inf. degree in computer and electrical engineering and the Dr.-Ing. degree in electrical engineering from the Technical University (TU) Dortmund, Dortmund, Germany, in 2005 and 2012, respectively. During 2006-2013, he was a Research Associate with the Institute of Control Theory and Systems Engineering, TU Dortmund. In 2013-2015, he was with Nagoya

Institute of Technology, Nagoya, Japan, where he was a specially appointed Assistant Professor. In 2015 he was an Associate Professor with the Department of Electrical Engineering, Nagaoka University of Technology, Nagaoka, Japan, before joining the University of Agder, Grimstad, Norway. $\mathrm{He}$ is a member of Faculty of Engineering and Sciences at the University of Agder. His current research interests include precise motion control, nonlinear systems with memory, flexible robots, and smart material actuators. Dr. Ruderman served as a Member of the Technical Program Committee for the IEEE Industrial Electronics Society (IES) 2014 International Workshop on Motion Control (AMC2014), the 2014 International Power Electronics Conference (IPEC2014), and the 2015 International Conference on Mechatronics (ICM2015) and as a Publication Co-chair for IEEE IES ICM2015. $\mathrm{He}$ is a Member of the Technical Committee on Motion Control and the Technical Committee on Sensors and Actuators of the IEEE IES. He is also Associate Editor of IFAC Mechatronics journal.
Makoto Iwasaki (Member) received the B.S., M.S., and Dr.Eng. de-

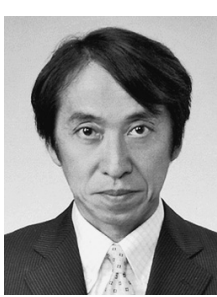
grees from Nagoya Institute of Technology, Nagoya, Japan, in 1986, 1988, and 1991, respectively, all in electrical and computer engineering. Since 1991, he has been with the Department of Computer Science and Engineering, Nagoya Institute of Technology, where he is currently a Professor. He was a Japanese Government Research Fellow with Chemnitz University of Technology, Chemnitz, Germany, from 1997 to 1998 and from 2002 to 2003 and with the Technical University of Munich, Munich, Germany, from 1997 to 1998. As professional society contributions of IEEE, he has been an elected Administrative Committee Member of IEEE/Industrial Electronics Society in term of 2010 to 2016, a Technical Editor for IEEE/ASME Trans on Mechatronic in term of 2010 to 2014, an Associate Editor for IEEE Trans on IE since 2014, a chair of Technical Committee on Motion Control of IEEE/IES, respectively. He also served as a general chair of IEEE/IES ICM2015. His current research interests are the applications of motion control theory and soft computing techniques for motor/motion control. Dr. Iwasaki is a member of The Institute of Electrical Engineers of Japan and the Japan Society for Precision Engineering. 